\title{
IRRIGACIÓN ARTERIAL DEL MIEMBRO PÉLVICO DE LA ALPACA (Vicugna pacos)
}

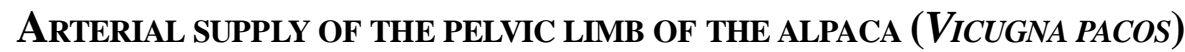

José Angulo A. ${ }^{1}$, Alberto Sato S. ${ }^{1,2}$, Miluska Navarrete Z. ${ }^{1}$, Jannet Cisneros S. ${ }^{1}$

\section{RESUMEN}

El objetivo de este trabajo fue identificar y describir los vasos arteriales del miembro pélvico de la alpaca (Vicugna pacos). Se utilizaron seis alpacas adultas ( 3 hembras y 3 machos). En la disección no se observaron diferencias anatómicas entre machos y hembras. La irrigación se originó a partir de la arteria ilíaca externa, principalmente, y de la arteria ilíaca interna. La arteria ilíaca externa fue el gran conducto arterial que se extendió por las diferentes regiones del miembro pélvico cambiando de denominación (femoral, poplítea, tibial craneal, dorsal del pie), desprendiendo múltiples ramas colaterales durante su curso. Las arterias circunfleja ilíaca superficial, circunfleja femoral lateral, safena y caudales femorales proximal y distal nacieron de la arteria femoral al igual que la arteria circunfleja femoral medial. La alpaca, en coincidencia con la llama, no presentó la arteria femoral profunda que se describe en el canino, equino y bovino. La arteria poplítea dio origen a la arteria descendente de la rodilla, como primera rama colateral, y a las arterias surales y otras ramas que se distribuyeron en la rodilla. La arteria tibial craneal, más larga, y la arteria tibial caudal, menos desarrollada, siguieron una distribución muy similar a la descrita en la llama. A nivel del tarso, la arteria safena se dividió en arteria plantar lateral y plantar medial. En el pie, la arteria plantar medial tuvo una mayor presencia anatómica, siendo su aporte vital para la perfusión sanguínea digital, dado el poco desarrollo de las arterias dorsal del pie y plantar lateral, como también ocurre en la llama. Así, aunque en el miembro pélvico de la alpaca se han observado varias arterias homólogas descritas en los textos clásicos de la anatomía de los animales domésticos, estas siguieron mayormente un patrón arterial similar a la llama.

Palabras clave: irrigación, alpaca, arterias, animales domésticos

\section{Abstract}

The aim of this work was to identify and describe the arterial vessels of the pelvic member of the alpaca (Vicugna pacos). Six adult alpacas were dissected ( 3 females and 3 males). There were no anatomical differences between males and females. The arterial

\footnotetext{
${ }^{1}$ Laboratorio de Anatomía Animal y Fauna Silvestre, Facultad de Medicina Veterinaria, Universidad Nacional Mayor de San Marcos, Lima

${ }^{2}$ E-mail: albertosato2000@yahoo.com
} 
blood supply leading to the pelvic limbs mainly originates from the external iliac artery and to a lesser extent from the internal iliac artery, as described also for the canine, equine, bovine and llama. The external iliac artery is the main arterial channel that runs through the different regions of the pelvic limb, changing the name (femoral, popliteal, cranial tibial, dorsal pedal), giving off several collateral branches during its course. The superficial circumflex iliac, lateral circumflex femoral, saphenous, the proximal and distal caudal femoral arteries arose from the femoral artery as does the medial circumflex femoral artery. In the alpaca, coinciding with the llama, did not present the deep femoral artery as described in other domestic animals such as dog, horse and bovine. The popliteal artery gives off to the descending genicular artery, as the first collateral branch, the sural arteries and other branches that were distributed at the knee. The longer cranial tibial artery and the less developed caudal tibial have a distribution very similar to that described in the llama. At the level of the tarsus, the saphenous artery divides into the lateral plantar and medial plantar arteries. In the hindfoot, the medial plantar artery has a greater anatomical presence, being its contribution vital to the digital blood perfusion, as occurs in the llama. Thus, although in the pelvic limb of the alpaca has been observed several homologous arteries described in the classic texts of anatomy of domestic animals, they mostly followed an arterial pattern similar to that of the llama.

Key words: irrigation, alpaca, arteries, domestic animals

\section{INTRODUCCIÓN}

Los camélidos sudamericanos (CSA) constituyen la mayor riqueza pecuaria y genética para los pobladores andinos (Fernández-Baca, 2005) y actualmente su crianza constituye una de las actividades de mayor importancia e impacto en el desarrollo socioeconómico de las comunidades campesinas del altiplano andino del Perú (Huanca, 2008). De esta ganadería dependen 2.9 millones de habitantes (el 12\% de la población nacional) (Moya y Torres, 2008).

Existen aproximadamente ocho millones de CSA en el mundo (Foronda et al., 2004), siendo el Perú el único país donde es posible encontrar poblaciones de las cuatro especies de camélidos (Pinto et al., 2010). Las especies domésticas, alpaca y llama, constituyen la mayor proporción, estimándose una población de 3780806 cabezas de alpacas y de 1262782 de llamas (INEI, 2010).

La alpaca, además de producir carne de alto valor nutritivo, posee una fibra de fina textura, que es muy apreciada en los merca- dos internacionales, donde cerca del $80 \%$ de la oferta mundial de esta fibra procede del Perú (Brenes et al., 2001), manteniéndose así como el principal productor de fibra de alpaca (De los Ríos, 2006). Por otro lado, la llama destaca por su mayor tamaño y fortaleza, y es útil como animal de carga en las áreas rurales (Iñiguez y Alem, 1996). Asimismo, las heces de ambos camélidos se emplean como combustible y fertilizante (Pinto et al., 2010). Actualmente se manifiesta un renovado interés en el estudio de la alpaca a fin de lograr potenciar un manejo sustentable, el cual está afianzado por las características productivas, su gran trascendencia económica y por ser un animal de muy bajo impacto ecológico (DESCO, 2006).

Con el fin de lograr el conocimiento integral de esta especie, y siguiendo el criterio de Arboleda-Sepúlveda (1986) y San Martín (1994) de elaborar información y documentación científica en forma sistemática y completa, se debe continuar el estudio de los aspectos fundamentales de la anatomía de la alpaca como parte de las ciencias básicas veterinarias. Esto permitirá sentar bases definidas de su conformación, ampliando el do- 
minio de su morfología y los horizontes científicos al aportar resultados aplicables a otras ciencias médicas veterinarias como la fisiología, patología, cirugía, radiología, y clínica, entre otras.

El nivel de conocimiento de la anatomía de la alpaca es aún incompleto. En la revisión de los textos anatómicos especializados sobre el miembro pélvico de la alpaca, se dispone de trabajos de investigación realizados en osteología (Fuentes, 1953), vasos linfáticos (Carrasco, 1968) y sistema muscular (Latínez, 1990). En esos trabajos se manifiesta la carencia o escasa profundidad en la información sobre otros aspectos anatómicos como es en el caso de estudios angiológicos arteriales, cuyas fuentes bibliográficas consultadas, no satisfacen plenamente el conocimiento exigido y detallado del abastecimiento arterial en el miembro posterior en esta especie. Además, cabe señalar que la mayor parte de la información anátomo-morfológica del sistema arterial del miembro pélvico en CSA ha sido generada en llamas.

Por tal motivo, el presente estudio tuvo el propósito de lograr un adecuado conocimiento de las características anatómicas en el cual se pueda identificar, describir y discutir la arborización del miembro pélvico de la alpaca.

\section{MateRiales y Métodos}

El presente trabajo se realizó en el Laboratorio de Anatomía Animal de la Facultad de Medicina Veterinaria, Universidad Nacional Mayor de San Marcos, Lima. El estudio de los especímenes se desarrolló entre octubre de 2009 y enero de 2010. Se emplearon seis alpacas Huacaya adultas, de ambos sexos (3 machos y 3 hembras), procedentes de la comunidad de San Felipe, departamento de Huancavelica, Perú. Los animales se encontraban en aparente buen estado de salud y habían sido descartados del rebaño para su venta al camal.
Los animales fueron anestesiados con pentobarbital sódico (Halatal®), vía endovenosa, en dosis, según Fowler (2010), de $20 \mathrm{mg} / \mathrm{kg}$ de peso vivo. Luego de alcanzado el plano anestésico profundo, se practicó una incisión longitudinal en el rafe medio ventral del cuello para exponer la arteria carótida común y la vena yugular externa. Se realizó un corte de $1 \mathrm{~cm}$ en ambos vasos a fin de obtener una sangría a blanco. Luego de la muerte del animal, se insertó una cánula en la arteria carótida común y se aplicó una solución conservadora a base de formol al 12\%, ácido fénico 3\%, glicerina al $8 \%$ y agua csp $100 \%$. Para este proceso, se usó una bomba compresora a una presión de $100 \mathrm{~mm}$ de $\mathrm{Hg}$, hasta lograr el retorno por la vena yugular externa. Se dejó reposar durante 6-8 días para la fijación de los tejidos y órganos, y luego se inyectó una solución de colorante a base de cola de carpintero granulada y anilina roja a través de la cánula insertada en la arteria carótida común.

La disección y exposición de las arterias se realizó desde el plano superficial al profundo, de proximal a distal y de craneal a caudal, considerando la división de las regiones topográficas en el miembro pélvico, así como la descripción de estructuras anatómicas según la terminología precisada por la Nómina Anatómica Veterinaria de la International Committee on Veterinary Gross Anatomical Nomenclature (ICVGAN) (2005).

\section{Resultados y Discusión}

El suministro sanguíneo de la extremidad pélvica derecha e izquierda de la alpaca se desarrolló a partir de las respectivas arterias ilíacas externa e interna, ramas terminales de la aorta abdominal, resultado que coincide a lo descrito por varios autores sobre el desarrollo de la irrigación del miembro pélvico en el canino (Miller et al., 1964), equino (Getty et al., 1990; Budras et al., 2009), bovino (Getty et al., 1990; Budras y Habel, 
2003) y llama (Graziotti et al., 1998, 2003). En la alpaca, la arteria ilíaca externa emergió desde la arteria aorta abdominal, a nivel de la extremidad craneal de la sexta vértebra lumbar. Por su parte, la arteria ilíaca interna surgió caudalmente a la arteria precedente cerca de la extremidad caudal de la misma vértebra lumbar (Fig. 1).

Dado su gran calibre y extensa prolongación regional, la arteria ilíaca externa representó la principal arteria para la irrigación del miembro pélvico en la alpaca, en forma similar a la llama (Graziotti et al., 1997) y, en general, a la de otros animales domésticos (Shively, 1993; König et al., 2005) (Fig. 1).

\section{Región de la Cintura Pélvica}

En la región de la cintura pelviana (región conformada por los huesos coxales articulados en el sínfisis pélvica), la arteria ilíaca interna en la alpaca dio origen a la arteria glútea craneal antes de que termine bifurcándose en las arterias glútea caudal y pudenda interna, en concordancia a lo señalado para la llama (Graziotti et al., 2003) y el bovino (Getty et al., 1990; Budras y Habel, 2003). Este hecho difiere con lo descrito en el canino (Miller et al., 1964) y en el equino (Getty et al., 1990), ya que en estas dos especies, la aparición de la arteria glútea craneal se produce desde la arteria glútea caudal como una

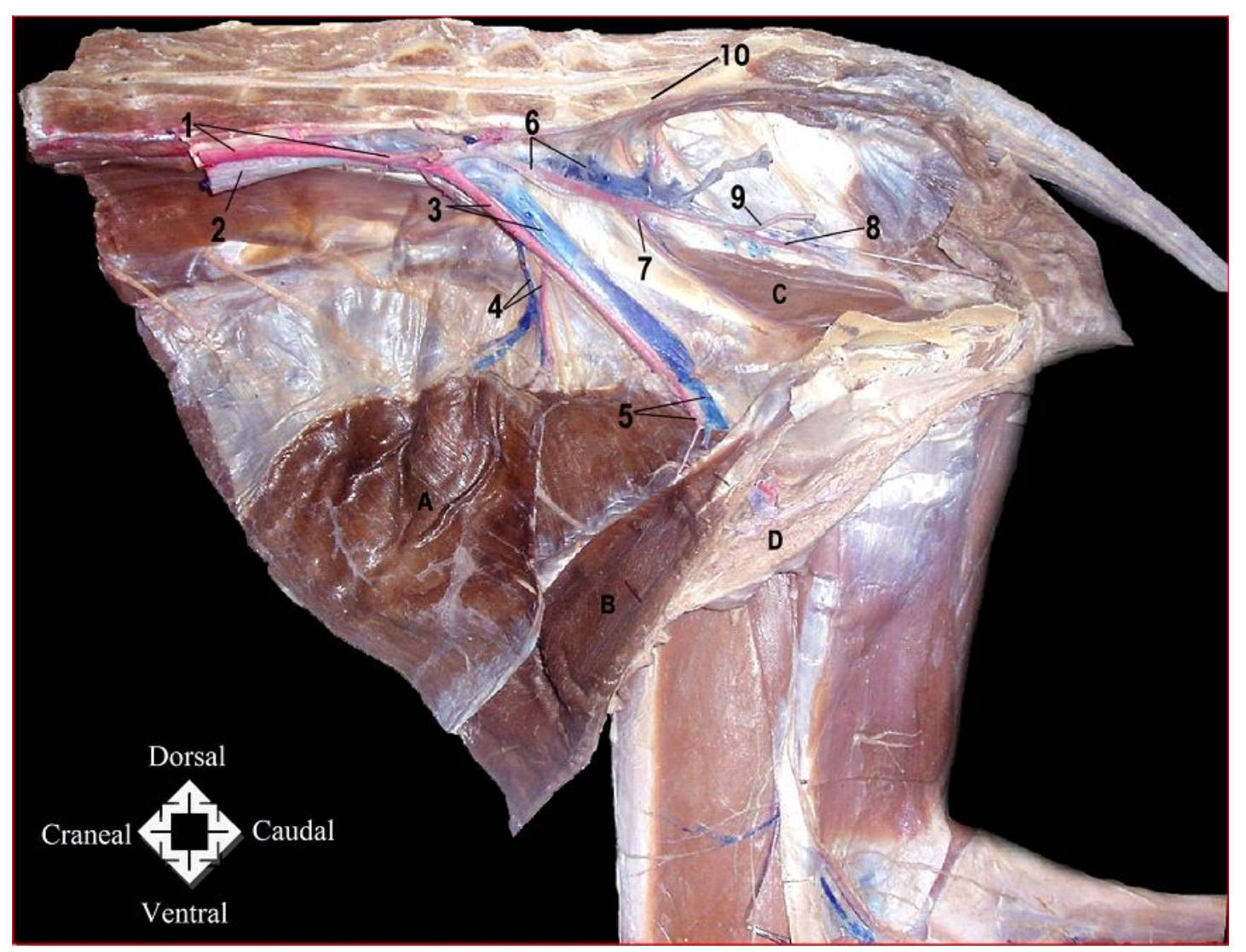

Figura 1. Vista medial derecha de la cavidad abdominal caudal y cavidad pélvica de una alpaca hembra. 1: Arteria aorta abdominal; 2: Vena cava caudal; 3: Arteria y vena ilíaca externa; 4: Arteria y vena circunfleja ilíaca profunda; 5: Tronco pudendoepigástrico; 6: Arteria y vena ilíaca interna; 7: Arteria obturadora; 8: Arteria glútea caudal; 9: Arteria pudenda interna; 10: Arteria sacra mediana; A: Músculo transverso del abdomen; B: Músculo recto abdominal; C: Músculo obturador interno; D: Glándula mamaria 
rama colateral adicional. Aún así, la contribución de esta arteria en la irrigación de la musculatura glútea de la alpaca fue similar a la encontrada en la llama y demás especies domésticas mencionadas. Asimismo, la arteria ilíaca interna, también dio origen desde un tronco común a unas delgadas arterias iliolumbar y obturadora, muy próximas a la arteria glútea craneal. El origen de estas arterias sólo concordó con lo señalado en la llama; esto es, por medio de un tronco común desde la arteria ilíaca interna (Graziotti et al., 2003) (Fig. 1).

\section{Región del Muslo}

\section{Arteria femoral}

Representó la continuación directa de la arteria ilíaca externa. Después de abandonar la cavidad abdominal a través de la laguna vascular se dirigió a la porción libre del miembro pélvico. El descenso en el triángulo femoral de la arteria femoral estuvo cubierto por el músculo sartorio, similar a lo observado en la llama (Graziotti et al., 1997) y el bovino (Shively, 1993; Budras y Habel, 2003), más no así en el equino y el canino donde cursa caudalmente al mismo (Shively, 1993).

A lo largo de su recorrido en la cara medial del muslo estuvo acompañada cranealmente por el nervio safeno y caudalmente por la vena femoral, descansando sobre el músculo iliopsoas y el músculo vasto medial, hasta alcanzar el canal aductor entre el tercio medio y distal del muslo, abandonando el triángulo femoral. Este canal fue formado por el músculo vasto medial y el tendón de inserción del músculo aductor. Inmediatamente a su salida del canal aductor, la arteria se ubicó entre las dos cabezas del músculo gastrocnemio en un plano profundo, continuando a través de este músculo como arteria poplítea (Fig. 2).

Las ramas colaterales que dio origen la arteria femoral durante su recorrido en la región del muslo fueron las siguientes:

\section{a) Arteria circunfleja ilíaca superficial}

Se originó en el tercio proximal del muslo, muy cerca o a partir de un tronco común con la arteria circunfleja femoral lateral, observándose que su origen y distribución es semejante a la arteria circunfleja ilíaca superficial señalada en la llama (Graziotti et al., 1997) y el canino (Miller et al., 1964; Getty et al., 1990; Shively, 1993). Se extendió proyectando dos ramas principales: una rama craneal y otra rama descendente (Fig. 2).

\section{b) Arteria circunfleja femoral lateral}

Surgió junto con la arteria circunfleja ilíaca superficial. Pasó cranealmente entre las cabezas del músculo cuadríceps (entre el músculo recto femoral y el músculo vasto medial), desprendiendo pequeños vasos hacia el músculo coxal articular y la cápsula articular de la cadera. Se dividió poco después en dos importantes ramas: la rama ascendente y la rama descendente. Esta última rama se bifurcó después de un corto recorrido enviando un vaso medialmente que se introdujo entre el músculo vasto intermedio y el músculo recto femoral (Fig. 2). En general, la distribución de esta arteria presenta mayor concordancia con lo descrito para la llama (Graziotti et al., 1997), salvo que se observó dos ramas transversas desde el vaso medial que desprendió la rama descendente, las cuales se anastomosaron con la arteria circunfleja femoral medial. En el canino, equino y bovino también se cita esta anastomosis entre las arterias circunflejas femorales lateral y medial aunque con ciertas diferencias respecto al modo origen de la rama transversa (Getty et al.,1990; König et al., 2005).

\section{c) Arteria circunfleja femoral medial}

Arteria colateral gruesa y profunda, originada directamente de la arteria femoral, diferente a lo descrito en textos clásicos de anatomía donde la arteria ilíaca externa, cuando aún está en el abdomen, emite la arteria femoral profunda que da origen al tronco 


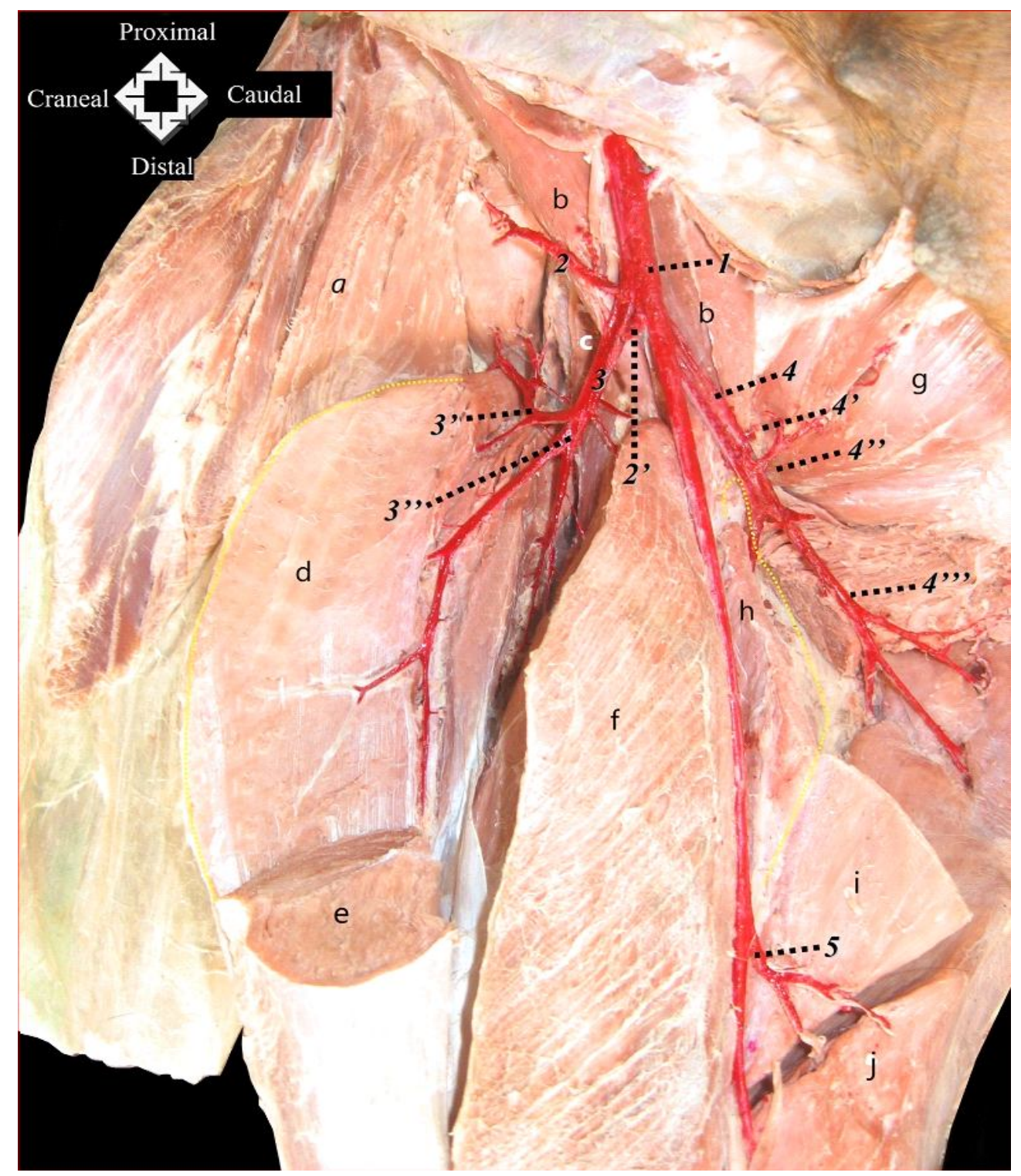

Figura 2. Vista craneomedial profunda del muslo derecho de la alpaca. 1: Arteria femoral; 2: A. circunfleja ilíaca superficial, rama craneal; 2': A. circunfleja ilíaca superficial, rama descendente (seccionada); 3: Arteria circunfleja femoral lateral; 3': Arteria circunfleja femoral lateral, rama ascendente; 3": Arteria circunfleja femoral lateral, rama descendente; 4: Arteria circunfleja femoral medial; 4': Arteria circunfleja femoral medial, rama acetabular; 4": Arteria circunfleja femoral medial, rama obturadora; 4"': Arteria circunfleja femoral medial, rama ascendente; 5: Arteria caudal femoral proximal; a: Músculo tensor de la fascia lata (seccionado); b: Músculo iliopsoas; c: Músculo articular coxal; d: Músculo cuadríceps (vasto lateral); e: Músculo cuadríceps (recto femoral, seccionado); f: Músculo cuadríceps (vasto medial); g: Músculo obturador externo; h: Músculo pectíneo (seccionado); $i$ : Músculo aductor (seccionado); $j$ : Músculo semimembranoso 
pudendoepigástrico para continuar como arteria circunfleja femoral medial, tal y como se presenta en el canino, equino y bovino (Budras y Habel, 2003, 2009; König et al., 2005). Este hallazgo en la alpaca también fue encontrado en la llama por Graziotti et al. (1997). Por otro lado, la arborización de la arteria en la alpaca fue similar a la señalada para la llama (Graziotti et al., 1997), canino, equino y bovino (Schaller, 2007), proporcionando la rama acetabular, rama ascendente, rama obturadora, rama profunda y rama transversa (Fig. 2).

\section{d) Arterias femorales caudales proximal $y$ distal}

Se conoce que el canino (Miller et al., 1964; Getty et al., 1990) y la llama (Graziotti et al., 1997) presentan tres arterias caudales femorales: proximal, media y distal. A diferencia de estas especies, la alpaca presentó sólo la arteria caudal femoral proximal y la distal. En la alpaca, la arteria femoral caudal proximal nació a partir del borde caudal de la arteria femoral, proximal al origen de la arteria safena y cubierta por fibras del músculo grácil (M. gracilis, recto interno). Presentó dos ramas importantes; una de ellas penetró el músculo grácil, músculo aductor y músculo semimembranoso. La otra rama se introdujo en el músculo semimembranoso abarcando territorios a cargo de la arteria caudal femoral media ausente en esta especie (Fig. 2).

La arteria femoral caudal distal surgió proximal a la arteria descendente de la rodilla a nivel del canal aductor y se dirigió hacia los músculos caudales de la región del muslo y de la pierna. Emitió una rama ascendente que se internó en la cara profunda del músculo bíceps femoral y una rama descendente que se extendió caudalmente sobre las cabezas del músculo gastrocnemio, enviando una rama proximal entre el músculo bíceps femoral y el músculo semitendinoso, irrigando a estos músculos y al nódulo linfático poplíteo. La rama descendente continuó su recorrido internándose entre las dos cabezas del músculo gastrocnemio (Fig. 3).

\section{e) Arteria safena}

Surgió a nivel de la inserción del músculo pectíneo, justo antes de que la arteria femoral comience a abandonar el triángulo femoral. En su origen fue cubierta por fibras tendinosas del músculo pectíneo y músculo grácil, continuando después sobre este último músculo, dirigiéndose superficialmente a la cara caudomedial de la pierna. Dio origen en su segmento proximal a una rama subcutánea que cursa cranealmente y a la rama articular de la rodilla, que se dirigió hacia el extremo proximal de la articulación de la rodilla, pero cubierta por fibras tendinosas del músculo sartorio (Fig. 3).

\section{f) Arteria descendente de la rodilla}

Surgió por dentro del canal aductor, distalmente a la arteria caudal femoral distal, arteria que sirve de referencia para hallar el origen de la arteria poplítea en los animales domésticos (Shively, 1993). A diferencia de la alpaca, en el canino, equino y bovino la arteria descendente de la rodilla surge proximal a la arteria caudal femoral distal (Miller et al., 1964; Getty et al., 1990; Shively, 1993). En la alpaca, el origen de la arteria descendente de la rodilla en relación a la arteria caudal femoral distal se produce distalmente a esta última. Por lo tanto, la arteria descendente de la rodilla nació como primera rama colateral de la arteria poplítea, y de acuerdo a observaciones en la llama (Graziotti et al., 1997, 1998) (Fig. 3).

\section{Región de la Pierna}

\section{Arteria poplitea}

La arteria poplítea fue la continuación directa de la arteria femoral en la región de la pierna. En su origen, abandonó el canal aductor y se introdujo entre las cabezas del músculo gastrocnemio; continuó a lo largo de la superficie flexora de la articulación de la rodilla y más distalmente fue cubierta por el músculo poplíteo en la cara caudal de la pierna (Fig. 3). A medida que descendió, emitió 


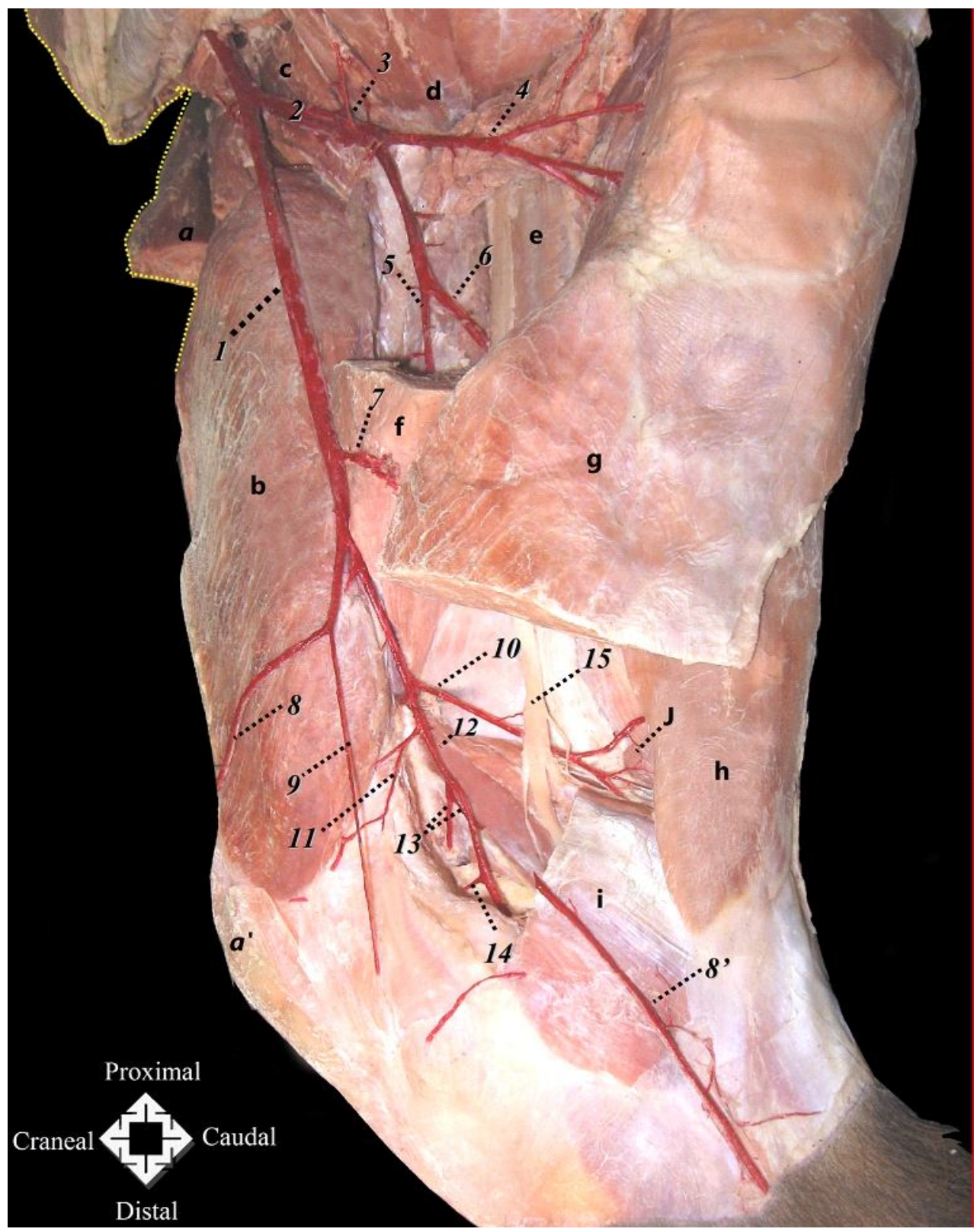

Figura 3. Vista caudomedial profunda del muslo y pierna derecha de la alpaca. 1: Arteria femoral; 2: Arteria circunfleja femoral medial; 3: Arteria circunfleja femoral medial, rama obturadora; 4: Arteria circunfleja femoral medial, rama ascendente; 5: Arteria circunfleja femoral medial, rama profunda; 6: Arteria circunfleja femoral medial, rama transversa; 7: Arteria caudal femoral proximal; 8: Arteria safena (seccionada y reflejada cranealmente); 8': Arteria safena (seccionada, continuación); 9: Arteria articular de la rodilla; 10: Arteria caudal femoral distal; 11: Arteria descendente de la rodilla; 12: Arteria poplitea; 13: Arterias surales; 14: Arteria media de la rodilla; 15: Nervio isquiático; a: Músculo tensor de la fascia lata (seccionado); b: Músculo cuadríceps (vasto medial); c: Músculo iliopsoas; d: Músculo obturador; e: Músculo bíceps femoral (cara profunda); $f$ : Músculo aductor (seccionado); g: Músculo semimembranoso (seccionado); $h$ : Músculo semitendinoso; $i$ : Músculo gastrocnemio (cabeza medial, seccio nado); j: Nódulo linfático poplíteo 


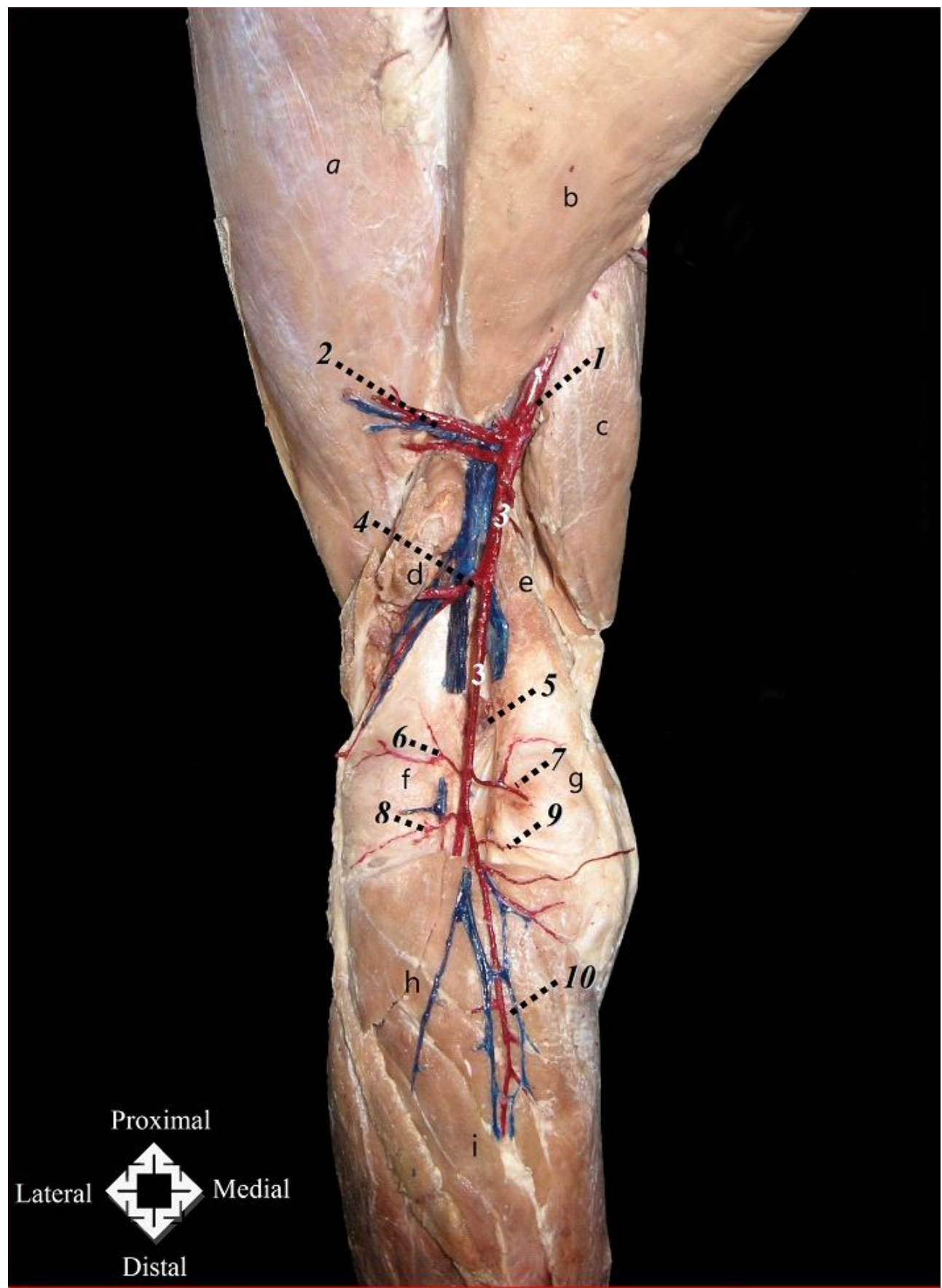

Figura 4. Vista caudal profunda de la rodilla izquierda de la alpaca. 1: Arteria femoral; 2: Arteria femoral caudal distal; 3: Arteria poplítea; 4: Arterias surales; 5; Arteria media de la rodilla; 6: Arteria proximal lateral de la rodilla; 7: Arteria proximal medial de la rodilla; 8: Arteria distal lateral de la rodilla; 9: Arteria distal medial de la rodilla; 10: Arteria tibial caudal; a: Músculo cuadríceps (vasto lateral); b: Músculo aductor; c: Músculo cuadríceps (vasto medial); d: Músculo gastrocnemio (cabeza lateral retirada); e: Músculo gastrocnemio (cabeza medial retirada); $f$ : Cóndilo lateral del fémur; g: Cóndilo medial del fémur; $h$ : Músculo poplíteo; $i$ : Músculo flexor digital profundo (cabeza medial) 

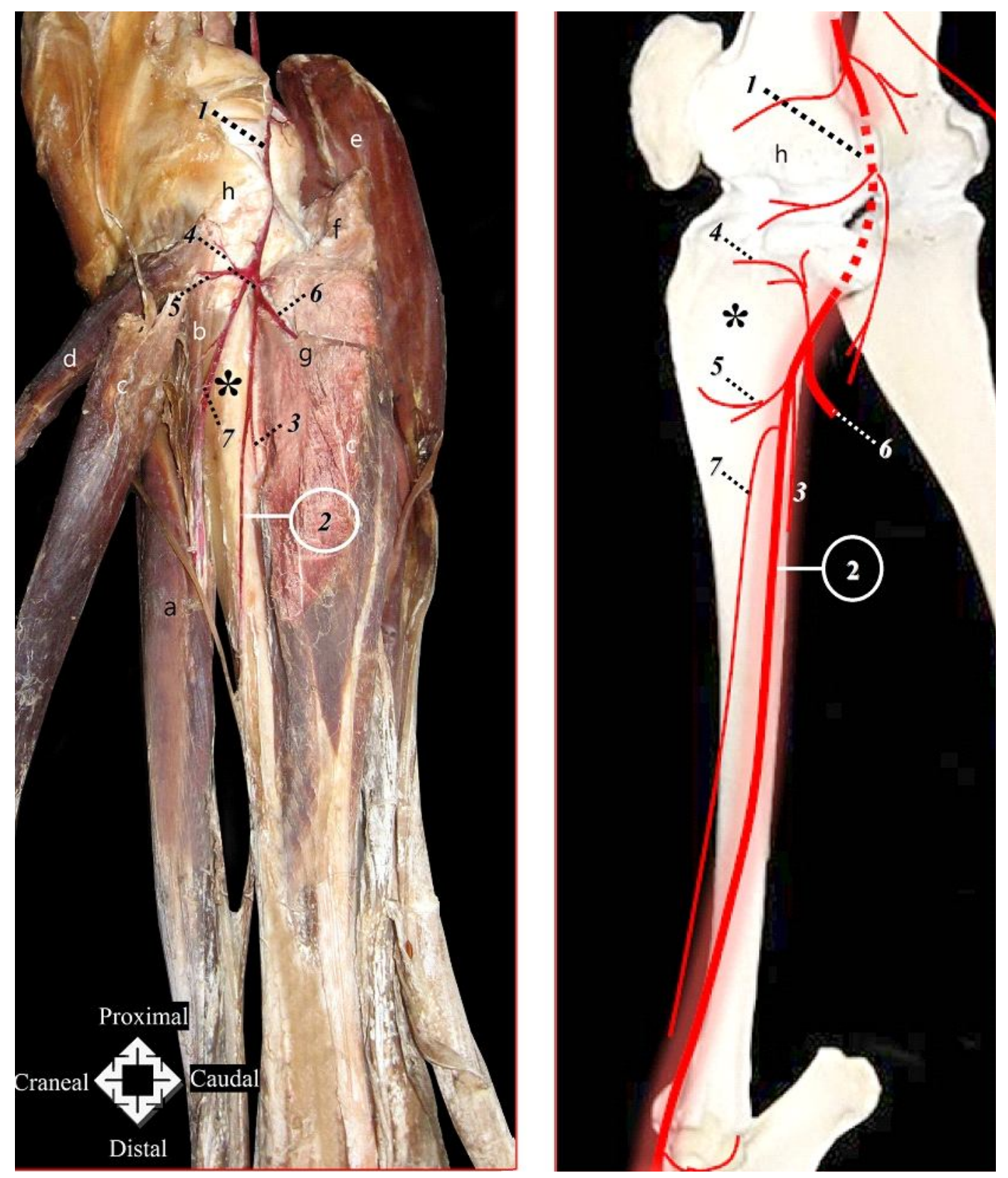

Figura 5. Vista lateral profunda y esquema ilustrativo de la pierna izquierda de la alpaca. 1 : Arteria poplítea; 2: Arteria tibial craneal; 3: Arteria nutricia de la tibia; 4: Arteria recurrente tibial craneal; 5: Rama transversal sobre el músculo tibial craneal; 6: Rama caudal hacia el músculo flexor profundo de los dedos; 7: Rama superficial; a: Músculo del tercer peróneo; b: Músculo extensor largo de los dedos; c: Músculo tibial craneal; d: Músculo peróneo largo; e: Músculo extensor lateral de los dedos; f: Músculo gastrocnemio (cabeza lateral seccionada); g: Músculo poplíteo (seccionado); $h$ : Músculo flexor profundo de los dedos; i: Cóndilo lateral de la tibia; *: Tibia 

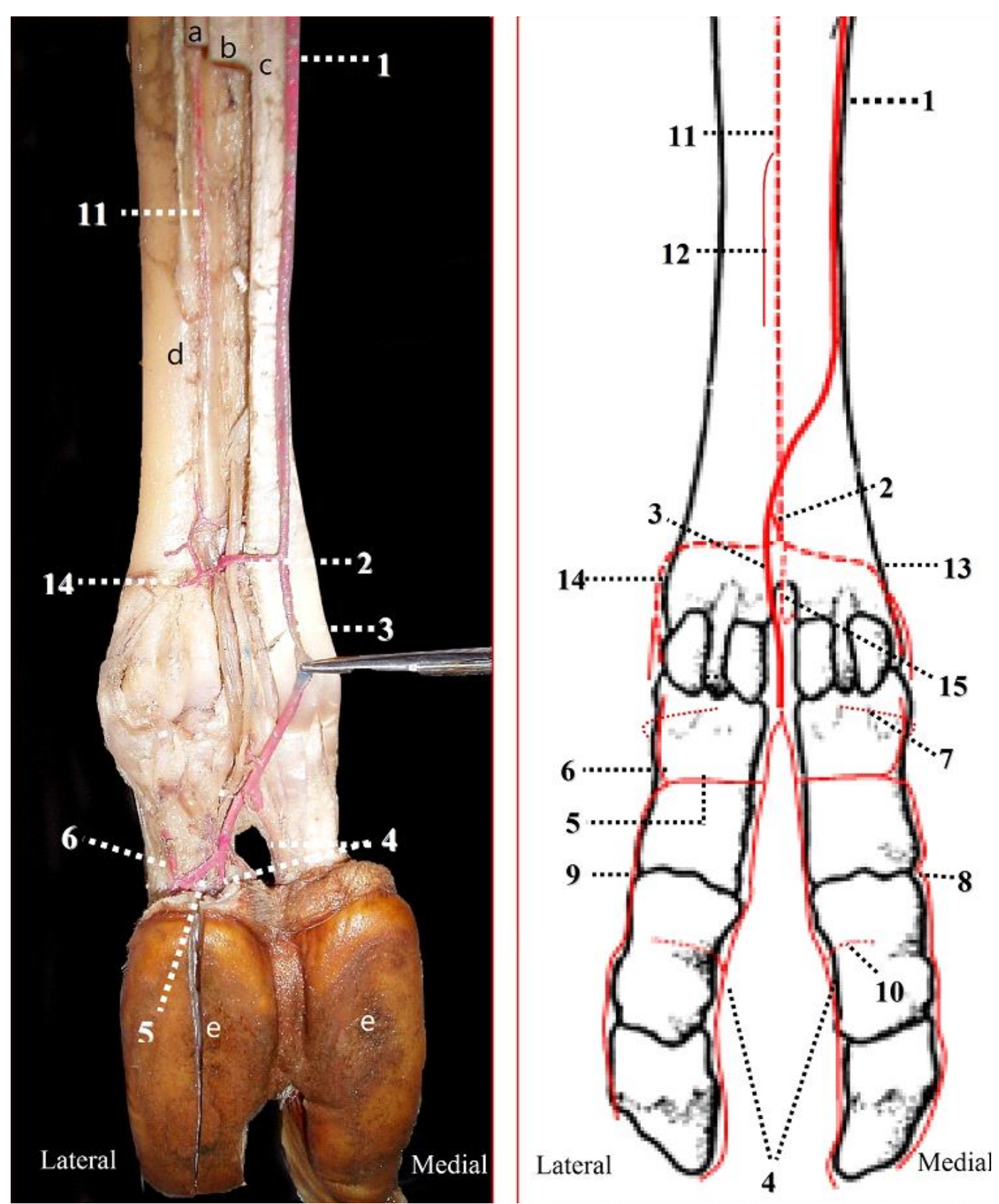

Figura 6. Vista plantar y esquema ilustrativo de las arterias del pie izquierdo de la alpaca. 1 : Arteria plantar medial, rama superficial; 2: Rama anastomótica; 3: Arteria digital común plantar III; 4: Arterias digitales propias III y IV axiales; 5: Rama plantar de la falange proximal; 6: Rama recurrente; 7: Rama dorsal de la falange proximal; 8: Arteria digital plantar propia III abaxial; 9: Arteria digital plantar propia IV abaxial; 10: Rama dorsal de la falange media; 11: Arteria metatarsiana plantar III; 12: Arteria nutricia del metatarso; 13: Arteria digital común plantar II; 14: Arteria digital común plantar IV; 15: Arteria metatarsiana perforante distal III; a: Músculo interóseo (seccionado); b: Músculo flexor profundo de los dedos (seccionado); c: Músculo flexor superficial de los dedos; d: Metatarso III y IV; e: Cojinetes plantares 
varias ramas para la musculatura de la pierna y la irrigación de la rodilla, las cuales fueron las arterias surales, la arteria media de la rodilla, las arterias proximales lateral y medial de la rodilla y la arteria distal lateral de la rodilla (Figs. 3 y 4 ).

En la alpaca la arteria distal medial de la rodilla surgió desde la arteria tibial caudal y no desde la arteria poplítea como en la llama (Graziotti et al., 1998), canino, equino y bovino (Schaller et al., 2007). Por lo demás, las otras arterias mencionadas en la alpaca provinieron desde la arteria poplítea al igual que las otras especies citadas, a excepción del bovino en el cual la arteria proximal femoral lateral surge desde la arteria femoral caudal.

Finalmente, la arteria poplítea, continuó por debajo del músculo poplíteo enviando ramas finas innominadas que se dirigieron a la cápsula de la rodilla, ligamentos cruzados y músculo popliteo. En el extremo proximal de la tibia, se orientó en sentido lateral para alcanzar la superficie craneolateral de la pierna atravesando, previamente, el espacio interóseo para continuar como arteria tibial craneal.

\section{Arteria safena (continuación)}

En la alpaca, así como en otras especies domésticas, descendió subcutáneamente junto con una vena satélite y el nervio safeno en la cara medial de la región de la pierna, resultando ser así la rama más extensa de la arteria femoral. En el canino (Miller et al., 1964; Getty et al., 1990) y en el equino (Getty et al., 1990; Shively, 1993) se divide en ramas craneal y caudal, pero esto no ocurre en la alpaca, donde la arteria desciende superficialmente, sin dividirse, concordando con el bovino (Getty et al., 1990; Shively, 1993) y la llama (Graziotti et al., 1998). Sin embargo, la arteria se insinuó caudalmente siguiendo un curso similar a la rama caudal, siendo entonces así considerada en estas dos últimas especies, según lo señalado por Getty et al. (1990) e ICVGAN (2005) en el bovino y Graziotti et al. (1998) en la llama. A medida que descendió a lo largo de la cara medial de la pierna, emitió varias ramas subcutáneas (Fig. 3).

En el tercio distal de la pierna, originó un vaso profundo de pequeño calibre que cursó craneolateralmente transversal al eje de la tibia, alcanzando al músculo tibial craneal y músculo extensor largo de los dedos. Luego, la arteria safena, a nivel del maléolo medial, emitió la arteria maleolar caudal lateral, que trazó un curso transverso al eje óseo sobre la cara caudal de la tibia dirigiéndose al maléolo lateral. Durante su recorrido, esta arteria emitió medialmente una rama calcánea que ascendió paralelamente a la tuberosidad calcánea y que a nivel de la expansión a manera de «capuchón» del tendón del músculo flexor superficial de los dedos (galea calcanea) proporcionó finos vasos que se distribuyeron en medial y lateral del calcáneo. Asimismo, un vaso arterial recurrente derivó de la rama calcánea y perforó la fascia que se halla entre el tendón calcáneo común y el músculo flexor profundo de los dedos.

La arteria safena después de dar origen a la arteria maleolar caudal lateral continuó en la cara medioplantar del tarso y se dividió en dos ramas terminales, las arterias plantares lateral y medial.

En general, la distribución de la arteria safena recuerda a la descripción referida a la arteria safena en la llama (Graziotti et al., 1998) existiendo una mayor similitud con ramas colaterales emitidas durante su curso, como la arteria maleolar caudal lateral, entre otras.

\section{Arteria tibial craneal}

Fue la continuación de la arteria poplítea y de mucho mayor longitud que la arteria tibial caudal. Describió un recorrido oblicuo lateral, pasando por debajo del origen de las tres cabezas del músculo flexor profundo de los 
dedos, atravesando luego, la parte proximal del espacio interóseo, para ubicarse cranealmente a la cinta fibrosa vestigial (representa el cuerpo de la fíbula), relacionándose al borde lateral de la tibia.

Profundamente, la arteria tibial craneal proporcionó las siguientes ramas principales: arteria nutricia de la tibia, arteria recurrente tibial craneal que ascendió sobre la superficie lateral de la tibia hacia el cóndilo lateral, una rama transversa sobre el músculo tibial craneal, una gruesa rama caudal penetrando el músculo flexor lateral de los dedos y la rama superficial que se extendió a lo largo de la superficie craneolateral de la tibia (Fig. 5). Finalmente, la arteria tibial craneal cursó debajo del retináculo extensor proximal y de los tendones de los músculos extensores hasta la cara dorsal del tarso continuando a partir de este punto como arteria dorsal del pie.

Teniendo en cuenta el recorrido particular de la arteria tibial craneal relacionado al borde lateral de la tibia en la alpaca, a diferencia del canino, equino y bovino que tiene una proyección más craneal a lo largo de la tibia; así como de la distribución de sus diferentes ramas generadas, se logró resultados más semejantes a los descritos en la llama (Graziotti et al., 1998), dado que presentó algunas ramas colaterales que sólo pudieron corresponderse con las halladas en la llama y con diferencias que aparecieron con algunas ramas descritas también en el canino y bovino (la rama superficial, por ejemplo), y la ausencia de otras en el equino.

\section{Arteria tibial caudal}

Se originó a partir de la arteria poplítea extendiéndose por encima del borde proximal del músculo poplíteo. Tuvo un escaso desarrollo en el miembro pélvico de la alpaca, hecho que explicaría el surgimiento de la arteria maleolar caudal lateral a partir de la arteria safena (que en equinos nace desde la propia arteria tibial caudal, mucho más desarrollada), tal como se describe en la llama (Graziotti et al., 1998). Sin embargo, durante su recorrido emitió la arteria distal medial de la rodilla y otras finas ramas para el músculo gastrocnemio y el músculo poplíteo (Fig. 4).

\section{Región del Pie}

La arteria dorsal del pie fue la continuación directa de la arteria tibial craneal, descendiendo en línea recta entre los tendones de los músculos tercer peróneo y extensor largo de los dedos sobre la cara dorsal del tarso emitiendo distal a la articulación tarsocrural las arterias tarsianas mediales y lateral. Finalmente, la arteria dorsal del pie continuó como una ramita escasamente desarrollada, la arteria metatarsiana dorsal III, que cursó dentro del surco longitudinal dorsal desapareciendo tras un corto trayecto.

En la cara plantar del pie, la arteria plantar lateral, rama terminal de la arteria safena, fue de corto trayecto y escaso diámetro, pasando por debajo del tendón del músculo flexor superficial de los dedos continuando como un vaso de fino calibre. Proporcionó ramas calcáneas que ascendieron por la tuberosidad del calcáneo que contribuyeron a formar la red calcánea.

A diferencia de la irrigación del pie para otros animales domésticos como en el canino, equino y bovino, donde la continuación distal de la arteria dorsal del pie y la arteria plantar lateral contribuyen a la misma en varios segmentos del pie (Getty et al., 1990), en la alpaca, el escaso desarrollo de estas dos arterias es suplida por la arteria plantar medial, que como segunda rama terminal procedente de la bifurcación de la arteria safena, fue de grosor y prolongación mucho más amplio proveyendo el flujo sanguíneo más importante del pie. Esta arteria fue indispensable en la formación del arco plantar profundo y en la anastomosis distal que dio origen a las arterias digitales (Fig. 6). Su distribución hasta donde se pudo observar en la alpaca se ajusta más a los resultados obtenidos en los estudios sobre las arterias del pie de la llama (Graziotti et al., 2000), presentando diferencias importantes con el canino, 
equino y bovino (Getty et al., 1990; Shively, 1993; König et al., 2005), respecto al origen y ramificación de varias arterias involucradas.

\section{Conclusiones}

- El aporte sanguíneo para el miembro pélvico de la alpaca provino principalmente de la arteria ilíaca externa, resultado comparable con otras especies de animales domésticos.

- La alpaca no presentó la arteria femoral profunda, hecho que es señalado también en la llama.

- La arteria circunfleja femoral medial se originó directamente de la arteria femoral ante la ausencia de la arteria femoral profunda hallazgo, que es descrito también en la llama.

- En la alpaca, la arteria descendente de la rodilla se identificó como la primera rama colateral de la arteria poplítea, en semejanza con la llama.

- La arteria maleolar caudal lateral surgió a partir de la arteria safena, dado el escaso desarrollo de la arteria tibial caudal en el miembro pélvico de la alpaca, hecho que coincide a lo descrito en la llama.

- La arteria plantar medial, debido a su grosor y longitud, fue la principal arteria de la irrigación del pie de la alpaca, hallazgo anatómicamente similar a lo observado en la llama.

- No se encontraron diferencias anatómicas en la irrigación arterial del miembro pélvico entre alpacas macho y hembra.

- Los hallazgos anátomo-morfológicos del sistema arterial permiten afirmar que la irrigación arterial del miembro de la alpaca fue muy similar a la descrita en la llama.

\section{Literatura Citada}

1. Arboleda-Sepúlveda O. 1986. La información y documentación científica como apoyo a la investigación sobre los camélidos sudamericanos. Rev Camélidos Sudam 2: 2-12.

2. Brenes $E$, Madrigal K, Pérez, F, Valladares K. 2001. El clúster de los camélidos en Perú: Diagnóstico competitivo y recomendaciones estratégicas. $\mathrm{La}$ Garita (CR): Instituto Centroamericano de Administración de Empresas. [Internet]. [21 julio 2010]. Disponible en: http://www.caf.com/attach/4/default/ CamelidosPeru.pdf

3. Budras K, Habel R. 2003. Bovine anatomy: an illustrated text. Hannover, Germany: Schlütersche. $144 \mathrm{p}$.

4. Budras K, Sack WO, Röck S. 2009. Anatomy of the horse. $5^{\text {th }}$ ed. Hannover, Germany: Schlütersche. 208 p.

5. Carrasco A. 1968. Vasos y centros linfáticos superficiales del miembro pélvico de la alpaca (Lama pacos). Tesis de Médico Veterinario. Lima: Univ Nac Mayor de San Marcos. 24 p.

6. De los Ríos E. 2006. Producción textil de fibras de camélidos sudamericanos en el área altoandina de Bolivia, Ecuador y Perú. Bruselas: UNIDO. [Internet]. [14 octubre 2010]. Disponible en: https:// www.unido.org/file-storage/download/ ?file_id $=58563$

7. [DESCO] Centro de Estudios y Promoción del Desarrollo Programa Regional Sur. 2006. Informe de la convención para el desarrollo sostenible del sector de los camélidos sudamericanos. II Simposium Internacional de Investigaciones sobre camélidos sudamericanos. Arequipa.

8. Fernández-Baca S. 2005. Situación actual de los camélidos sudamericanos en Perú. Proyecto de Cooperación Técnica en apoyo de la crianza y aprovechamiento de los camélidos sudamericanos en la región Andina, TCP/RLA/2914. Roma: FAO. 63 p.

9. Foronda R, Luna O, Morales E. 2004. Perfil de proyecto oportunidades económicas para criadores de camélidos del municipio de Morochata. La Paz: FAO. $63 \mathrm{p}$. 
10. Fowler M. 2010. Medicine and surgery of camelids. $3^{\text {rd }}$. Iowa: Wiley-Blackwell. $636 \mathrm{p}$.

11. Fuentes L. 1953. Contribución a la osteología de la alpaca (Lama glama pacos). Tesis de Médico Veterinario. Lima: Univ Nac Mayor de San Marcos. 77 p.

12. Getty R, Sisson S, Grosman J. 1990. Anatomía de los animales domésticos. $5^{\circ}$ ed. Barcelona: Salvat. 2302 p.

13. Graziotti G, Ríos C, RodríguezMenéndez J, Victorica C. 1997. La irrigación arterial del muslo de la llama (Lama glama). Rev Chil Anat 15(1): 35-40.

14. Graziotti G, Ríos C, RodríguezMenéndez J, Victorica C. 1998. Irrigación de la rodilla y pierna de la llama (Lama glama). Rev Chil Anat 16(1): 89-94.

15. Graziotti G, Ríos C, RodríguezMenéndez J, Victorica C. 2000. Arteries of the hindfoot of the llama (Lama glama). Anat Histol Embryol 29: 273-276.

16. Graziotti G, Rodríguez-Menéndez J, Victorica C, Fux Solveyra G Ríos C. 2003. Systematic study of the internal iliac artery in llama (Lama glama). Ann Anat 185: 461-463.

17. Huanca T. 2008. Efecto de la administración de gonadotropinas exógenas (FSH y eCG) en la respuesta ovárica y la producción de embriones en alpacas (Vicugna pacos). Tesis doctoral. Galicia, España: Universidad de Santiago de Compostela. $142 \mathrm{p}$.

18. [ICVGAN] International Committee on Veterinary Gross Anatomical Nomenclature. 2005. Nómina Anatómica Veterinaria. $5^{\text {th }}$ ed. Hannover, Germany. $190 \mathrm{p}$

19. [INEI] Instituto Nacional de Estadística e Informática. 2010. Agropecuario. En: Junín: Compendio estadísti- co departamental 2009. Lima: Oficina Departamental de Estadística e Informática de Junín. p 417-448.

20. Iñiguez LC, Alem R. 1996. Role of camelids as means of transportation and exchange in the Andean region of Bolivia. World Anim Rev 86: 12-21.

21. König H, Rubert J, Liebich H. 2005. Órganos del sistema cardiovascular. En: König H, Liebich H (eds). Anatomía de los animales domésticos: Órganos, sistema circulatorio y sistema nervioso. $2^{\circ}$ ed. Tomo 2. Madrid: Médica Panamericana. p 153-188.

22. Latínez B. 1990. Contribución al estudio de los músculos del miembro pélvico de la alpaca (Lama pacos). Tesis de Médico Veterinario. Lima: Univ Nac Mayor de San Marcos. 42 p.

23. Miller M, Christensen G, Evans $H$. 1964. Anatomy of the dog. Philadelphia: WB Saunders. 941 p.

24. Moya E, Torres J. 2008. Familias alpaqueras enfrentando al cambio climático. Lima: Soluciones prácticasITDG. $107 \mathrm{p}$.

25. Pinto C, Martín C, Cid M. 2010. Camélidos sudamericanos: clasificación, origen y características. Rev Complutense Cienc Vet 4(1): 23-26.

26. San Martín F. 1994. Avances y alternativas de alimentación para los camélidos sudamericanos. Rev Inv Vet Perú 7(2). [Internet]. [06 abril 2009]. Disponible en: http://sisbib.unmsm.edu.pe/bvrevistas/veterinaria/v07_n2/avancesya.htm

27. Schaller 0.2007 . Illustrated veterinary anatomical nomenclature. $2^{\text {nd }}$ ed. Stuttgart, Germany: Enke Verlag. 615 p.

28. Shively M. 1993. Anatomía veterinaria básica, comparativa y clínica. México: Manual Moderno. 391 p. 